\title{
Hepatic Steatosis and Thyroid Function Tests in Overweight and Obese Children
}

\author{
L. Pacifico, ${ }^{1}$ E. Bonci, ${ }^{1}$ F. Ferraro, ${ }^{1}$ G. Andreoli, ${ }^{1}$ S. Bascetta, ${ }^{1}$ and C. Chiesa ${ }^{2}$ \\ ${ }^{1}$ Department of Pediatrics, Sapienza University of Rome, Viale Regina Elena, 32400161 Rome, Italy \\ ${ }^{2}$ Institute of Translational Pharmacology, National Research Council, 00133 Rome, Italy \\ Correspondence should be addressed to L. Pacifico; lucia.pacifico@uniromal.it
}

Received 30 October 2012; Revised 11 January 2013; Accepted 19 January 2013

Academic Editor: Carine Beysen

Copyright (C) 2013 L. Pacifico et al. This is an open access article distributed under the Creative Commons Attribution License, which permits unrestricted use, distribution, and reproduction in any medium, provided the original work is properly cited.

\begin{abstract}
Objectives. Associations between thyroid function and nonalcoholic fatty liver disease (NAFLD) are unknown in childhood. Thus, the aim of the present study was to investigate in 402 consecutive overweight/obese children the association between thyroid function tests and hepatic steatosis as well as metabolic variables. Methods. Hepatic steatosis was diagnosed by ultrasound after exclusion of infectious and metabolic disorders. Fasting serum samples were taken for determination of thyroid function (TSH, FT4, and FT3), along with alanine aminotransferase (ALT), lipid profile, glucose, insulin, and insulin resistance (IR). Results. Eighty-eight children (21.9\%) had TSH above the normal range (>4.0 mIU/L). FT3 and FT4 were within the reference intervals in all subjects. Elevated TSH was associated with increased odds of having hepatic steatosis (OR 2.10 (95\% CI, 1.22-3.60)), hepatic steatosis with elevated ALT (2.42 (95\% CI, 1.29-4.51)), hypertriglyceridemia, elevated total cholesterol, and IR as well as metabolic syndrome (considered as a single clinical entity), after adjustment for age, gender, pubertal status, and body mass index-SD score (or waist circumference). Conclusions. In overweight/obese children, elevated TSH concentration is a significant predictor of hepatic steatosis and lipid and glucose dysmetabolism, independently of the degree of total and visceral obesity.
\end{abstract}

\section{Introduction}

Over the last two decades, the rise in the prevalence rates of overweight and obesity may explain the emergence of nonalcoholic fatty liver disease (NAFLD) as the leading cause of liver disease worldwide [1]. NAFLD, a novel component of metabolic syndrome (MetS) [2], is a spectrum of fatassociated liver conditions that can result in end-stage liver disease and the need for liver transplantation $[1,3]$. Simple steatosis, or fatty liver, occurs early in NAFLD and may progress to nonalcoholic steatohepatitis (NASH), fibrosis, and cirrhosis with increased risk of hepatocellular carcinoma $[1,3]$.

Several previous studies have addressed the association between thyroid function and NAFLD [4-10]. The results of these studies support an association between overt or subclinical thyroid disease and NAFLD. This seems to be biologically plausible given the common clinical and biochemical features of central obesity, insulin resistance (IR), hypertension, hypertriglyceridemia, and lipid peroxidation observed in both NAFLD and hypothyroidism [5, 11]. However, confirmation and further characterization of clinical data supporting this association are needed. To our knowledge, none of the previous studies have investigated the relationship between hepatic steatosis and thyroid function in childhood. Because small abnormalities in thyroid function are common, thyroid function may importantly influence the prevalence of NAFLD in a population of overweight/obese children and adolescents. We therefore tested the hypothesis that even slightly elevated serum levels of TSH indicating mild thyroid failure might be associated with an increased risk for NAFLD. To this end we explored in overweight/obese children with no clinical, autoantibody, and ultrasonographic evidence of thyroid disease the association between thyroid function tests (thyroid-stimulating hormone (TSH), and free thyroxine (FT4), free triiodothyronine (FT3)) and hepatic steatosis as well as MetS and its parameters. 


\section{Materials and Methods}

2.1. Study Subjects. This is a cross-sectional study carried out at the Hepatology outpatient Clinic of the Department of Pediatrics, Sapienza University of Rome, Italy, between April 2010 and September 2012. The children included were all those being overweight (body mass index (BMI) higher than the age- and sex-specific 85th percentile) or obese (BMI higher than the age- and sex-specific 95th percentile) [12] aged 6-16 years. However, the following subjects were excluded: (a) those with renal disease; type 1 or 2 diabetes; any condition known to influence body composition, insulin action, or insulin secretion (e.g., glucocorticoid therapy, overt hypothyroidism, and Cushing's disease); (b) those with known thyroid disease, current or past history of thyroid hormone or antithyroid drug intake, thyroid alterations in volume and morphology at ultrasound, and positive antithyroglobulin (anti-TG) and anti-thyroperoxidase (anti-TPO) antibodies; (c) those with any laboratory or clinical evidence suggesting an alternate or coexistent underlying chronic liver disease including hepatic virus infections (Hepatitis A-E and G, cytomegalovirus, and Epstein-Barr virus), autoimmune hepatitis, metabolic hepatic disease, $\alpha$-1-antitrypsin deficiency, cystic fibrosis, Wilson's disease, hemochromatosis, and celiac disease; and (d) those with history of alcohol consumption and smoking (where appropriate).

All study participants underwent physical examination including measurements of weight, standing height, BMI, waist circumference (WC), determination of the stage of puberty, degree of obesity, and systolic blood pressure (BP) and diastolic BP. The pubertal stage was categorized into two groups (prepubertal: boys with pubic hair and gonadal stage I, and girls with pubic hair and breast stage I; pubertal: boys with pubic hair and gonadal stage $\geq \mathrm{II}$ and girls with pubic hair stage and breast stage $\geq \mathrm{II}$ ). WC was obtained at the midpoint between the lowest rib and the iliac crest. The measurement was made at the end of a normal expiration while the subjects were in a standing position. The degree of obesity was quantified using Cole's least mean-square method, which normalizes the skewed distribution of BMI and expresses $\mathrm{BMI}$ as a SD score (SDS). This measure gives age- and genderspecific estimates of the distribution median, the coefficient of variation, and the degree of skew by a maximum likelihood fitting technique [12]. Systolic and diastolic BP were measured twice at the right arm after a 10 min rest in the supine position by using an automated oscillatory system (Dinamap Vital Signs Monitor, Model 1846 SX; Criticon Incorporated, Tampa, FL, USA).

The study was approved by the Hospital Ethics Committee, and informed consent was obtained from subjects' parents prior to assessment.

2.2. Laboratory Investigations. Blood samples were taken from each subject, after an overnight fast, for estimation of glucose, insulin, total cholesterol and high-density lipoprotein cholesterol (HDL-C), triglycerides, alanine aminotransferase (ALT), gamma-glutamyl transferase (GGT), TSH, FT4, FT3, and anti-TG and anti-TPO antibodies. All analyses were conducted by COBAS 6000. TSH, FT3, FT4, anti-TG and anti-TPO antibodies, and insulin were measured on cobas e 601 module (Electrochemiluminescence Technology, Roche Diagnostics), while the remaining analytes on cobas c 501 clinical chemistry module (Photometric Technology), according to the instructions of the manufacturer.

TSH was considered normal if it was between 0.4 and $4.0 \mathrm{mIU} / \mathrm{L}$ [13-16]. The reference intervals for FT3 and FT4 were $2.70-5.20 \mathrm{pg} / \mathrm{mL}$ and $0.80-1.90 \mathrm{ng} / \mathrm{dL}$, respectively. Threshold values for anti-TG and anti-TPO antibodies were 115 and $34 \mathrm{IU} / \mathrm{mL}$, respectively.

2.3. Definitions. Ultrasound examinations of the liver and of the thyroid were performed in real time using an Aplio XV (Toshiba Medical Systems) with $3.5-5-\mathrm{MHz}$ convex transducers and tissue harmonics. Hepatic steatosis was defined by an appearance of hyperechoic liver parenchyma with tightly packed fine echoes and posterior beam attenuation. At ultrasound, thyroid size and morphology were evaluated with a high-resolution $7.5 \mathrm{MHz}$ linear transducer, with the subjects sitting and their necks slightly extended. Thyroid volume was defined as enlarged according to the reference values for age, gender, and body surface area [17]. Thyroid tissue echogenicity was evaluated in a longitudinal scan of the thyroid lobes by a standardized comparison with the echogenicity of the adjacent muscles: sternohyoideus, sternothyroideus, and sternocleidomastoideus. Ultrasound scans were performed by a well-trained ultrasonographist who was unaware of the clinical and laboratory data.

For the American Heart Association (AHA) [18], MetS is diagnosed in the presence of any three of the following five constituent risks: central obesity as determined by WC, hypertension, low HDL-C values, elevated triglyceride values, and glucose impairment. We used the pediatric AHA definition [19], which is based on the AHA adult definition but uses pediatric reference standards for BP, WC, triglycerides, and HDL-C. Thus, in our study, central obesity was defined as a WC $\geq 90$ th percentile for age and gender; hypertriglyceridemia as triglycerides $\geq 90$ th percentile for age and gender; low HDL-C as concentrations $\leq 10$ th percentile for age and gender; elevated BP as systolic or diastolic BP $\geq 90$ th percentile for age, gender, and height percentile; and impaired fasting glucose as glucose $\geq 5.6 \mathrm{mmol} / \mathrm{L}$. IR was determined by a homeostasis model assessment of insulin resistance (HOMA-IR) [20]. We considered HOMA-IR values $\geq 90$ th percentile for age and gender of those previously observed in a local population of healthy normal-weight children as an indicator of IR [21].

2.4. Statistical Analysis. Statistical analyses were performed using the SPSS package. Data are expressed either as frequencies or means with $95 \%$ confidence intervals (CIs). Distributions of continuous variables were examined for skewness and kurtosis and were logarithmically transformed, when appropriate. Geometric means are reported for TSH, FT3, FT4, total cholesterol and HDL-C, triglycerides, insulin, and HOMA-IR values. Differences between groups were tested for significance using independent sample $t$-test for quantitative variables, and chi-square test for qualitative 
TABLE 1: Baseline characteristics of 402 overweight/obese children according to serum TSH.

\begin{tabular}{|c|c|c|c|}
\hline & \multicolumn{2}{|c|}{ Serum TSH } & \multirow{2}{*}{$P$ value } \\
\hline & $\leq 4 \mathrm{mIU} / \mathrm{L}(n=314)$ & $>4 \mathrm{mIU} / \mathrm{L}(n=88)$ & \\
\hline TSH, mIU/L & $2.42(2.33-2.52)$ & $4.74(4.52-4.95)$ & $<0.0001$ \\
\hline Age, years & $10.7(10.3-11.1)$ & $10.9(10.0-11.2)$ & 0.46 \\
\hline Male gender, $n(\%)$ & $175(55.7)$ & $55(62.5)$ & 0.35 \\
\hline Prepubertal, $n(\%)$ & $159(50.6)$ & $58(65.9)$ & 0.56 \\
\hline Weight, Kg & $61(59-63)$ & $60(55-65)$ & 0.70 \\
\hline Height, cm & $149(147-152)$ & $149(144-153)$ & 0.80 \\
\hline BMI, $\mathrm{Kg} / \mathrm{m}^{2}$ & $26.3(25.7-26.8)$ & $26.3(25.2-27.3)$ & 0.75 \\
\hline BMI-SDS & $2.0(1.94-2.2)$ & $2.0(1.9-2.2)$ & 0.80 \\
\hline Waist circumference, $\mathrm{cm}$ & $87(84-90)$ & $89(87-90)$ & 0.26 \\
\hline Systolic blood pressure, $\mathrm{mmHg}$ & $109(107-110)$ & $110(107-112)$ & 0.88 \\
\hline Diastolic blood pressure, $\mathrm{mmHg}$ & $69(68-70)$ & $70(67-72)$ & 0.82 \\
\hline Triglycerides, mg/dL & $99(90-108)$ & $119(103-135)$ & $<0.01$ \\
\hline Total cholesterol, mg/dL & $160(156-165)$ & $176(166-186)$ & $<0.01$ \\
\hline $\mathrm{HDL}-\mathrm{C}, \mathrm{mg} / \mathrm{dL}$ & $47(46-49)$ & $45(43-48)$ & 0.30 \\
\hline Fasting glucose, $\mathrm{mmol} / \mathrm{L}$ & $4.71(4.64-4.78)$ & $4.67(4.58-4.75)$ & 0.48 \\
\hline Insulin, mU/L & $16.0(15.0-18.0)$ & $19.3(16.5-22.0)$ & $<0.05$ \\
\hline HOMA-IR values & $3.62(3.22-4.0)$ & $4.0(3.4-4.6)$ & $<0.05$ \\
\hline ALT, IU/L & $31(28-35)$ & $52(36-68)$ & $<0.01$ \\
\hline GGT, IU/L & $17(16-19)$ & $22(18-26)$ & $<0.01$ \\
\hline FT3, pg/mL & $4.26(4.18-4.35)$ & $4.35(4.26-4.42)$ & 0.14 \\
\hline FT4, ng/dL & $1.18(1.15-1.22)$ & $1.18(1.12-1.22)$ & 0.76 \\
\hline
\end{tabular}

Data are expressed as $n$ (\%), mean or geometric mean (95\% confidence intervals).

TSH: thyroid-stimulating hormone; BMI: body mass index; BMI-SDS: BMI-SD score; HDL-C: high-density lipoprotein cholesterol; HOMA-IR: homeostasis model assessment of insulin resistance; ALT: alanine aminotransferase; GGT: gamma-glutamyl transferase; FT3: free triiodothyronine; FT4: free thyroxine.

variables. Pearson's correlation and linear regression coefficients were used to examine the relationship between variables. Logistic regression analysis was performed to determine the independence of the association of TSH levels with hepatic steatosis, hepatic steatosis with elevated ALT, and metabolic variables, after adjustments for age, gender, pubertal status, and BMI-SDS (or WC) as well as FT3 and FT4 concentrations. Relationships between TSH levels and hepatic steatosis were also examined after adjustment for anthropometric and metabolic variables including hypertriglyceridemia, hypercholesterolemia, and insulin resistance. In the fully adjusted regression model, FT3 and FT4 levels were also included. A $P$ value of less than 0.05 was considered statistically significant.

\section{Results}

3.1. Clinical and Laboratory Features of Study Population. The study group consisted of a consecutive series of 402 children fulfilling the selection criteria. Ninety-seven and 305 were overweight and obese, respectively. In Table 1 clinical, metabolic, and hormonal characteristics of overweight/obese children according to TSH level are summarized. Eightyeight subjects $(21.9 \%)$ had TSH above the normal range $(>4.0 \mathrm{mIU} / \mathrm{L})$. FT3 and FT4 were within the reference intervals in all subjects.
Compared to individuals with normal TSH, subjects with hyperthyrotropinemia showed significantly higher ALT, GGT, total cholesterol, triglycerides, insulin, and HOMA-IR values. Children with TSH $\leq 4.0 \mathrm{mIU} / \mathrm{L}$ and those with TSH over the normal range did not significantly differ in values of free thyroid hormones as well as in age, gender, pubertal status, or degree of total and central adiposity.

3.2. Thyroid Function Tests in relation to NAFLD and Metabolic Variables. Serum TSH concentrations were significantly higher in the 144 children with fatty liver compared to the 258 children with no fatty liver (3.20 (95\% CI, 2.95-3.36) versus 2.65 (95\% CI, 2.51-2.80); $P<0.01$ ). The children with hepatic steatosis did not differ significantly from those with no hepatic steatosis in respect of FT3 (4.35 (95\% CI, 4.264.48) versus 4.30 (95\% CI, 4.14-4.40)) and FT4 (1.17 (95\% CI, $1.13-1.21)$ versus 1.21 (95\% CI, 1.17-1.24)).

As shown in Table 2, elevated TSH concentrations were significantly associated with an increased prevalence of hepatic steatosis, and hepatic steatosis with elevated ALT concentrations (serum ALT $>25$ IU/L for boys and $>22$ IU/L for girls [22]). Among the metabolic variables, the prevalence of hypertriglyceridemia, IR, elevated total cholesterol, and MetS (considered as a single clinical entity) was significantly higher in the 88 subjects with elevated TSH concentrations than in the 314 subjects with normal TSH concentrations. 
TABLE 2: Prevalence of hepatic steatosis, hepatic steatosis with elevated ALT, metabolic syndrome, and its components according to serum TSH among 402 overweight/obese children.

\begin{tabular}{|c|c|c|c|}
\hline & \multicolumn{2}{|c|}{ Serum TSH } & \multirow{2}{*}{$P$ value } \\
\hline & $\leq 4 \mathrm{mIU} / \mathrm{L}(n=314)$ & $>4 \mathrm{mIU} / \mathrm{L}(n=88)$ & \\
\hline Hepatic steatosis & $101(32.2)$ & $43(48.9)$ & $<0.01$ \\
\hline Hepatic steatosis with elevated ALT & $73(23.2)$ & $31(35.2)$ & $<0.01$ \\
\hline Central obesity & $148(47.1)$ & $41(46.6)$ & 0.58 \\
\hline Elevated blood pressure & $23(7.3)$ & $6(6.8)$ & 0.78 \\
\hline Hypertriglyceridemia & $69(22.0)$ & $35(39.8)$ & $<0.01$ \\
\hline Low HDL-C & $57(18.2)$ & $17(19.3)$ & 0.20 \\
\hline Elevated total cholesterol & $94(29.9)$ & $39(44.3)$ & $<0.01$ \\
\hline Glucose $\geq 5.6 \mathrm{mmol} / \mathrm{L}$ & $8(2.5)$ & $2(2.3)$ & 0.35 \\
\hline Insulin resistance & $89(28.3)$ & $39(44.3)$ & $<0.01$ \\
\hline Metabolic syndrome & $32(10.2)$ & $17(19.3)$ & $<0.05$ \\
\hline
\end{tabular}

Data are presented as $n(\%)$.

TSH: thyroid-stimulating hormone; HDL-C: high-density lipoprotein cholesterol; ALT: alanine aminotransferase.

TABLE 3: Adjusted odds ratios (and 95\% CIs) of hepatic steatosis, hepatic steatosis with elevated ALT, metabolic syndrome, and its components among 402 overweight/obese children.

\begin{tabular}{|c|c|c|c|}
\hline & \multicolumn{2}{|c|}{ Serum TSH } & \multirow{2}{*}{$P$ value } \\
\hline & $\leq 4 \mathrm{mIU} / \mathrm{L}(n=314)$ & $>4 \mathrm{mIU} / \mathrm{L}(n=88)$ & \\
\hline \multicolumn{4}{|l|}{ Hepatic steatosis } \\
\hline Adjusted OR $(95 \% \mathrm{CI})^{+, *}$ & 1.00 (referent) & $2.10(1.22-3.60)$ & $<0.01$ \\
\hline \multicolumn{4}{|c|}{ Hepatic steatosis with elevated ALT } \\
\hline Adjusted OR (95\% CI) $)^{+, *}$ & 1.00 (referent) & $2.42(1.29-4.51)$ & $<0.01$ \\
\hline \multicolumn{4}{|l|}{ Central obesity } \\
\hline Adjusted OR $(95 \% \mathrm{CI})^{+}$ & 1.00 (referent) & $1.32(0.57-3.01)$ & 0.52 \\
\hline \multicolumn{4}{|l|}{ Elevated blood pressure } \\
\hline Adjusted OR $(95 \% \mathrm{CI})^{+, *}$ & 1.00 (referent) & $1.31(0.41-4.17)$ & 0.58 \\
\hline \multicolumn{4}{|l|}{ Hypertriglyceridemia } \\
\hline Adjusted OR $(95 \% \mathrm{CI})^{+, *}$ & 1.00 (referent) & $2.58(1.39-4.78)$ & $<0.01$ \\
\hline \multicolumn{4}{|l|}{ Low HDL-C } \\
\hline Adjusted OR $(95 \% \mathrm{CI})^{+, *}$ & 1.00 (referent) & $1.97(0.98-3.98)$ & 0.063 \\
\hline \multicolumn{4}{|l|}{ Elevated total cholesterol } \\
\hline Adjusted OR $(95 \% \mathrm{CI})^{+, *}$ & 1.00 (referent) & $2.23(1.30-3.84)$ & $<0.01$ \\
\hline \multicolumn{4}{|l|}{ Glucose $\geq 5.6 \mathrm{mmol} / \mathrm{L}$} \\
\hline Adjusted OR $(95 \% \mathrm{CI})^{+, *}$ & 1.00 (referent) & 0 & \\
\hline \multicolumn{4}{|l|}{ Insulin resistance } \\
\hline Adjusted OR $(95 \% \mathrm{CI})^{+, *}$ & 1.00 (referent) & $2.62(1.45-4.74)$ & $<0.01$ \\
\hline \multicolumn{4}{|l|}{ Metabolic syndrome } \\
\hline Adjusted OR $(95 \% \mathrm{CI})^{+, *}$ & 1.00 (referent) & $2.55(1.13-5.76)$ & $<0.05$ \\
\hline
\end{tabular}

Table 3 shows the multivariate-adjusted associations between TSH and hepatic steatosis, as well as metabolic variables. High TSH was associated with an increased odds of having hepatic steatosis, hepatic steatosis with elevated ALT, hypertriglyceridemia, elevated total cholesterol, and IR as well as MetS, after adjustment for age, gender, pubertal status, and BMI-SDS (or WC) as well as FT3 and FT4. When a stepwise multivariate regression analysis included variables such as age, gender, pubertal status, BMI-SDS, hypertriglyceridemia, hypercholesterolemia, IR, and FT3 and FT4, the association between hepatic steatosis and TSH levels remained statistically significant (OR, 1.96 (95\% CI, 1.10-3.75); $P<0.05)$. In this model, other covariates independently associated with hepatic steatosis were hypertriglyceridemia (2.73 (95\% CI, 1.51-4.92); $P<0.01)$ and IR (2.37 (95\% CI, 1.38-4.06); $P<$ $0.01)$. 
No association was found between FT4 and hepatic steatosis as well as MetS and its parameters after controlling for age, gender, pubertal status, and BMI-SDS (or WC).

\section{Discussion}

To the best of our knowledge, our study is the first to assess the relationships between thyroid function and hepatic steatosis in a large consecutive series of young participants. First, we found that in overweight/obese children and adolescents hyperthyrotropinemia was significantly associated with hepatic steatosis, after adjustments for age, gender, pubertal status, and degree of obesity. Second, we also found a significant association between elevated TSH levels and metabolic variables (including hypertriglyceridemia and IR which are usually altered in fatty liver). Finally, multivariate regression analysis showed that mild abnormalities in thyroid function were associated with hepatic steatosis independently of metabolic risk factors.

Previous studies have investigated the association between NAFLD (or surrogate markers of NAFLD) and thyroid function but solely in adult or elderly patients [4$10,23]$. The study by Targher et al. involving a large cohort of unselected adult outpatients was the first to report a strong association between thyroid function tests and serum liver enzyme activity concentrations [23]. In particular, Targher et al. found a significant positive relationship between serum TSH, ALT, and GGT activities throughout the normal and high TSH ranges, and a similar inverse relationship between FT4 and serum liver enzyme activity concentrations [23]. Notably, these results did not change after adjustment for a broad spectrum of potential confounders, such as gender, age, fasting glucose and lipid parameters. Unfortunately, that study did not provide information on medication use, lifestyle characteristics (i.e., daily alcohol consumption), obesity status, and degree of insulin resistance among study participants. The recent data from a large-scale populationbased study [9], the Study of Health in Germany (SHIP), partly agree with those from Targher et al. [23]. In the SHIP study, there was a significant inverse association between FT4 concentration and hepatic steatosis (defined by the presence of a hyperechogenic ultrasound pattern of the liver and increased ALT concentrations), whereas TSH and FT3 concentrations were not consistently associated with hepatic steatosis, thus suggesting that overt but not subclinical hypothyroidism (SH) might be associated with hepatic steatosis [9]. In a cross-sectional study performed among 878 euthyroid elderly Chinese who took their annual healthy examination, the prevalence rate of ultrasound-diagnosed NAFLD showed an increasing trend as serum TSH level increased, while the rate showed a decreasing trend as serum FT4 level increased [6]. Finally, in a recent cross-sectional study involving 4,648 health check-up subjects [7], Chung et al. found that the prevalence of ultrasound-diagnosed NAFLD and abnormal ALT levels increased steadily with increasing grades of hypothyroidism (for NAFLD, subclinical: $29.9 \%$ and overt: $36.3 \%$; for abnormal ALT, $20.1 \%$ and $25.9 \%, P<0.001$, resp.). SH was defined by Chung et al. as a serum TSH level over $4.1 \mathrm{mIU} / \mathrm{L}$ with normal FT4 concentration (0.7-1.8 ng/dL), while overt hypothyroidism as a FT4 level less than $0.7 \mathrm{ng} / \mathrm{dL}$. An $1 \mathrm{IU} / \mathrm{L}$ increase of natural logged TSH was associated with a $20 \%$ increase in the prevalence of NAFLD, independently of known risk factors such as age, gender, BMI, WC, triglyceride, HDL-C, hypertension, and diabetes [7].

The published literature investigating the association between thyroid function and NAFLD has also included patients with biopsy-proven NAFLD. Liangpunsakul and Chalasani retrospectively found that the prevalence of hypothyroidism was significantly higher in adult patients with NASH when compared with age-, gender-, race-, and body weight-matched adults without liver disease attending the general medical clinics [4]. In that study, however, data on thyroid function tests were not available and cases were defined solely based on use of synthetic T4 replacement therapy, therefore suggesting a high frequency of participants with overt hypothyroidism. Likewise, in a case control study involving 256 adult patients with NAFLD and 430 age-, gender-, race-, and BMI-matched adult outpatients with normal liver tests and no evidence of acute or chronic liver disease, Pagadala et al. retrospectively found that subjects with hypothyroidism were 2.1 (95\% CI, 1.1-3.9, $P=0.02$ ) and 3.8 (95\% CI, 2-6.9, $P<0.001$ ) times more likely to have NAFLD and NASH, respectively [8]. After adjusting for diabetes, hypertension, BMI, and hyperlipidemia, subjects with hypothyroidism were still found to be 2.1 times (95\% CI, $1.1-3.9, P=0.022$ ) more likely to have NAFLD than those without hypothyroidism. In addition, hypothyroidism was significantly higher in patients with NASH compared to those with no NASH [8]. However, that study again did not include laboratory data regarding thyroid function, and subjects were defined as having hypothyroidism if they carried a clinical diagnosis of hypothyroidism and were on thyroid replacement therapy. Yet, in a study involving 69 euthyroid adult patients with primary NAFLD [10], Carulli et al. retrospectively found that when biopsy-proven NASH patients were compared to those with no NASH, high TSH levels, independently of MetS and IR, were predictors of $\mathrm{NASH}$, but the possibility of selection bias was raised in that study as well as in the previous ones $[4,5,8]$ because of liver biopsy.

Against that background, the strengths of our study are the careful clinical characterization of the participants with their risk factor profiles, and by pediatric standards a fairly large study sample. Compared to previous studies [4-10, 23], our data differ in several design features including demographic and clinical characteristics (young versus adult or older participants with more advanced forms of the disease), inclusion and exclusion criteria, data collection (prospective versus retrospective), study selection (consecutive versus nonconsecutive series), population details (sufficient versus insufficient), study design (cohort versus case control), or participant recruitment (hospital-based versus populationbased study). Nonetheless, our results as a whole allow us to confirm and expand on the findings of previous reports. In a population of overweight/obese children and adolescents, even slightly elevated serum levels of TSH are associated with an increased risk for NAFLD. 
There are several underlying mechanisms which may substantiate the relationship between thyroid dysfunction and hepatic steatosis. Thyroid hormones influence all major metabolic pathways, and thyroid dysfunction, especially hypothyroidism, has been associated with IR [24, 25], dyslipidemia $[26,27]$, and obesity $[28,29]$, all of which play an important role in the development of NAFLD. IR in the setting of hypothyroidism has been documented [25] and is associated with decreased responsiveness of glucose uptake in muscle and adipose tissue to insulin, as well as decreased glycogen synthesis in skeletal muscle in both animal and human studies $[24,25,30,31]$. These effects were alleviated by thyroid replacement [24]. Recently, IR has been shown to also exist in $\mathrm{SH}$ and to be comparable to that of clinical hypothyroidism [32]. Another explanation for the association between thyroid function and NAFLD is that hypothyroidism is associated with abnormal lipid values [27]. Hypothyroidism primarily causes elevation in cholesterol and lowdensity lipoproteins but it also affects the synthesis, mobilization and degradation of all aspects of lipid metabolism [26]. The elevation of triglycerides in hypothyroid subjects is caused by a reduced removal rate of triglycerides from plasma due to a decrease in the activity of hepatic triglyceride lipase $[26,33]$. The effects of $\mathrm{SH}$ on serum lipids values are less clear. While some studies have demonstrated that serum triglycerides, lipid subparticle size, and low-density lipoprotein cholesterol (LDL-C) oxidizability may be altered in patients with $\mathrm{SH}$, others have not shown any effect on these lipid alterations [34]. In agreement with previous studies in children $[35,36]$, we found that elevated TSH was significantly associated with hypertriglyceridemia as well as IR, independent of total and central obesity. Finally, hypothyroidism has been reported to modulate mitochondrial nitric oxide synthesis and alter mitochondrial inner membrane composition and permeability which alters respiratory gene expression and mitochondrial oxygen uptake [37]. Such abnormalities would result in increased ADP concentration and generation of reactive oxygen species [38].

This study has a few potential limitations. First, we had no histopathological data. Nonetheless, liver biopsies in a large study collective such as our cohort are difficult to perform, also for ethical reasons, as no specific therapy follows histologic diagnosis of NAFLD apart from recommending lifestyle modification which is generally advised to all obese children. Conversely, ultrasonography is by far the most common way of diagnosing hepatic steatosis in clinical practice. Second, it is cross-sectional; thus, our data are associations and do not prove causality. As opposed to healthy controls, serum TSH concentrations have been consistently found to be higher in obese subjects $[13,39]$. Unlike TSH, data regarding the circulating levels of free thyroid hormones are discrepant between different studies, which reported either increased or decreased serum concentrations of FT3 [13, 28, 40, 41], with normal or decreased FT4/FT3 ratios $[28,41]$. Because in some studies TSH fell with weight loss [42], it has been suggested that higher TSH in obese subjects is simply a metabolic adaption to excess body fat. However, weight and/or fat loss does not predictably decrease TSH and T3 [43-45]. Others have suggested that obesity-related $\mathrm{SH}$, characterized by an increased serum TSH concentration with normal concentrations of the thyroid hormones, may be associated with dyslipidemia, IR, subclinical inflammation, and increased risk for coronary heart disease $[46,47]$. In the study by Aeberli et al. who prospectively examined the associations between changes in thyroid function, IR, and other metabolic risk factors in obese children undergoing weight and fat loss in a well-controlled 8-week inpatient program [35], changes in TSH did not correlate with losses of weight, fat, or lean tissue (as assessed by dual energy X-ray absorption), but they significantly correlated with fasting insulin and HOMA-IR, independently of body weight and body composition. Yet, in that study [35], at baseline variation of TSH, but not variations in body weight or body fat, was a significant predictor of triglycerides, total cholesterol, and LDL-C. Baseline TSH was also an independent predictor of fasting insulin and HOMAIR. In accordance with Aeberli et al. [35], we found that elevated TSH is a significant predictor of lipid and glucose dysmetabolism as well as of hepatic steatosis, independently of the degree of total and central obesity. Nonetheless, the possibility that thyroid hormones and NAFLD share common genetic or environmental influences accounting for the observed association cannot be discounted [48]. It is also possible that hepatic steatosis affects thyroid function rather than the other way around [48]. A longitudinal study with a cohort of children with and without hepatic steatosis at baseline would help clarify this.

\section{Conflict of Interests}

No potential conflict of interests relevant to this paper was reported.

\section{Acknowledgment}

This study was supported by a grant from Sapienza University of Rome (Progetti di Ricerca Universitaria 2010-2011).

\section{References}

[1] P. Angulo, "Nonalcoholic fatty liver disease," The New England Journal of Medicine, vol. 346, no. 16, pp. 1221-1231, 2002.

[2] A. Kotronen and H. Yki-Järvinen, "Fatty liver: a novel component of the metabolic syndrome," Arteriosclerosis, Thrombosis, and Vascular Biology, vol. 28, no. 1, pp. 27-38, 2008.

[3] L. Pacifico, E. Poggiogalle, V. Cantisani et al., "Pediatric nonalcoholic fatty liver disease: a clinical and laboratory challenge," World Journal of Hepatology, vol. 2, no. 7, pp. 275-288, 2010.

[4] S. Liangpunsakul and N. Chalasani, "Is hypothyroidism a risk factor for non-alcoholic steatohepatitis?" Journal of Clinical Gastroenterology, vol. 37, no. 4, pp. 340-343, 2003.

[5] M. G. Silveira, F. D. Mendes, N. N. Diehl, F. T. Enders, and K. D. Lindor, "Thyroid dysfunction in primary biliary cirrhosis, primary sclerosing cholangitis and non-alcoholic fatty liver disease," Liver International, vol. 29, no. 7, pp. 1094-1100, 2009.

[6] C. Xu, L. Xu, C. Yu, M. Miao, and Y. Li, "Association between thyroid function and nonalcoholic fatty liver disease in euthyroid elderly Chinese," Clinical Endocrinology, vol. 75, no. 2, pp. 240-246, 2011. 
[7] G. E. Chung, D. Kim, W. Kim et al., "Non-alcoholic fatty liver disease across the spectrum of hypothyroidism," Journal of Hepatology, vol. 57, no. 1, pp. 150-156, 2012.

[8] M. R. Pagadala, C. O. Zein, S. Dasarathy, L. M. Yerian, R. Lopez, and A. J. McCullough, "Prevalence of hypothyroidism in nonalcoholic fatty liver disease," Digestive Diseases and Sciences, vol. 57, no. 2, pp. 528-534, 2012.

[9] T. Ittermann, R. Haring, H. Wallaschofski et al., "Inverse association between serum free thyroxine levels and hepatic steatosis: results from the study of health in Pomerania," Thyroid, vol. 22, no. 6, pp. 568-574, 2012.

[10] L. Carulli, S. Ballestri, A. Lonardo et al., "Is nonalcoholic steatohepatitis associated with a high-though-normal thyroid stimulating hormone level and lower cholesterol levels?" Internal and Emergency Medicine. In press.

[11] C. J. Gardner, P. Richardson, C. Wong, N. Polavarapu, G. J. Kemp, and D. J. Cuthbertson, "Hypothyroidism in a patient with non-alcoholic fatty liver disease," British Medical Journal, vol. 342, no. 7789, pp. 168-170, 2011.

[12] T. J. Cole, M. C. Bellizzi, K. M. Flegal, and W. H. Dietz, "Establishing a standard definition for child overweight and obesity worldwide: international survey," British Medical Journal, vol. 320, no. 7244, pp. 1240-1243, 2000.

[13] L. Pacifico, C. Anania, F. Ferraro, G. M. Andreoli, and C. Chiesa, "Thyroid function in childhood obesity and metabolic comorbidity," Clinica Chimica Acta, vol. 413, no. 3-4, pp. 396405, 2012.

[14] T. E. Hamilton, S. Davis, L. Onstad, and K. J. Kopecky, “Thyrotropin levels in a population with no clinical, autoantibody, or ultrasonographic evidence of thyroid disease: implications for the diagnosis of subclinical hypothyroidism," Journal of Clinical Endocrinology and Metabolism, vol. 93, no. 4, pp. 1224-1230, 2008.

[15] G. Brabant, P. Beck-Peccoz, B. Jarzab et al., "Is there a need to redefine the upper normal limit of TSH?" European Journal of Endocrinology, vol. 154, no. 5, pp. 633-637, 2006.

[16] P. Laurberg, S. Andersen, A. Carlé, J. Karmisholt, N. Knudsen, and I. B. Pedersen, "The TSH upper reference limit: where are we at?" Nature Reviews Endocrinology, vol. 7, no. 4, pp. 232-239, 2011.

[17] M. B. Zimmermann, S. Y. Hess, L. Molinari et al., "New reference values for thyroid volume by ultrasound in iodinesufficient schoolchildren: A World Health Organization/Nutrition for Health and Development Iodine Deficiency Study Group Report," American Journal of Clinical Nutrition, vol. 79, no. 2, pp. 231-237, 2004.

[18] S. M. Grundy, "Metabolic syndrome scientific statement by the American Heart Association and the National Heart, Lung, and Blood Institute," Arteriosclerosis, Thrombosis, and Vascular Biology, vol. 25, no. 11, pp. 2243-2244, 2005.

[19] E. Goodman, S. R. Daniels, J. B. Meigs, and L. M. Dolan, "Instability in the diagnosis of metabolic syndrome in adolescents," Circulation, vol. 115, no. 17, pp. 2316-2322, 2007.

[20] D. R. Matthews, J. P. Hosker, A. S. Rudenski, B. A. Naylor, D. F. Treacher, and R. C. Turner, "Homeostasis model assessment: insulin resistance and $\beta$-cell function from fasting plasma glucose and insulin concentrations in man," Diabetologia, vol. 28, no. 7, pp. 412-419, 1985.

[21] L. Pacifico, C. Anania, F. Martino et al., "Functional and morphological vascular changes in pediatric nonalcoholic fatty liver disease," Hepatology, vol. 52, no. 5, pp. 1643-1651, 2010.
[22] J. B. Schwimmer, W. Dunn, G. J. Norman et al., "SAFETY study: alanine aminotransferase cutoff values are set too high for reliable detection of pediatric chronic liver disease," Gastroenterology, vol. 138, no. 4, pp. 1357-1364, 2010.

[23] G. Targher, M. Montagnana, G. Salvagno et al., "Association between serum TSH, free T4 and serum liver enzyme activities in a large cohort of unselected outpatients," Clinical Endocrinology, vol. 68, no. 3, pp. 481-484, 2008.

[24] C. Rochon, I. Tauveron, C. Dejax et al., "Response of glucose disposal to hyperinsulinaemia in human hypothyroidism and hyperthyroidism," Clinical Science, vol. 104, no. 1, pp. 7-15, 2003.

[25] G. Dimitriadis, P. Mitrou, V. Lambadiari et al., "Insulin action in adipose tissue and muscle in hypothyroidism," Journal of Clinical Endocrinology and Metabolism, vol. 91, no. 12, pp. 49304937, 2006.

[26] E. Pucci, L. Chiovato, and A. Pinchera, "Thyroid and lipid metabolism," International Journal of Obesity and Related Metabolic Disorders, vol. 24, supplement 2, pp. S109-S112, 2000.

[27] T. O’Brien, S. F. Dinneen, P. C. O’Brien, and P. J. Palumbo, "Hyperlipidemia in patients with primary and secondary hypothyroidism," Mayo Clinic Proceedings, vol. 68, no. 9, pp. 860866, 1993.

[28] M. A. Michalaki, A. G. Vagenakis, A. S. Leonardou et al., "Thyroid function in humans with morbid obesity," Thyroid, vol. 16, no. 1, pp. 73-78, 2006.

[29] Y. Raftopoulos, D. J. Gagné, P. Papasavas et al., "Improvement of hypothyroidism after laparoscopic Roux-en-Y gastric bypass for morbid obesity," Obesity Surgery, vol. 14, no. 4, pp. 509-513, 2004.

[30] G. Dimitriadis, M. Parry-Billings, S. Bevan et al., “The effects of insulin on transport and metabolism of glucose in skeletal muscle from hyperthyroid and hypothyroid rats," European Journal of Clinical Investigation, vol. 27, no. 6, pp. 475-483, 1997.

[31] G. D. Dimitriadis, B. Leighton, M. Parry-Billings, D. West, and E. A. Newsholme, "Effects of hypothyroidism on the sensitivity of glycolysis and glycogen synthesis to insulin in the soleus muscle of the rat," Biochemical Journal, vol. 15, no. 2, pp. 369$373,1989$.

[32] E. Maratou, D. J. Hadjidakis, A. Kollias et al., "Studies of insulin resistance in patients with clinical and subclinical hypothyroidism," European Journal of Endocrinology, vol. 160, no. 5, pp. 785-790, 2009.

[33] E. N. Pearce, "Hypothyroidism and dyslipidemia: modern concepts and approaches," Current Cardiology Reports, vol. 6, no. 6, pp. 451-456, 2004.

[34] E. N. Pearce, "Update in lipid alterations in subclinical hypothyroidism," Journal of Clinical Endocrinology and Metabolism, vol. 97, no. 2, pp. 326-333, 2012.

[35] I. Aeberli, A. Jung, S. B. Murer et al., "During rapid weight loss in obese children, reductions in TSH predict improvements in insulin sensitivity independent of changes in body weight or fat," Journal of Clinical Endocrinology and Metabolism, vol. 95, no. 12, pp. 5412-5418, 2010.

[36] N. S. Nader, R. S. Bahn, M. D. Johnson, A. L. Weaver, R. Singh, and S. Kumar, "Relationships between thyroid function and lipid status or insulin resistance in a pediatric population," Thyroid, vol. 20, no. 12, pp. 1333-1339, 2010.

[37] M. C. Carreras, J. G. Peralta, D. P. Converso et al., "Modulation of liver mitochondrial NOS is implicated in thyroid-dependent regulation of $\mathrm{O} 2$ uptake," American Journal of Physiology, vol. 281, no. 6, pp. H2282-H2288, 2001. 
[38] V. P. Skulachev, "Role of uncoupled and non-coupled oxidations in maintenance of safely low levels of oxygen and its oneelectron reductants," Quarterly Reviews of Biophysics, vol. 29, no. 2, pp. 169-202, 1996.

[39] M. Rotondi, P. Leporati, A. La Manna et al., "Raised serum TSH levels in patients with morbid obesity: is it enough to diagnose subclinical hypothyroidism?" European Journal of Endocrinology, vol. 160, no. 3, pp. 403-408, 2009.

[40] R. Sari, M. K. Balci, H. Altunbas, and U. Karayalcin, "The effect of body weight and weight loss on thyroid volume and function in obese women," Clinical Endocrinology, vol. 59, no. 2, pp. 258262, 2003.

[41] G. De Pergola, A. Ciampolillo, S. Paolotti, P. Trerotoli, and R. Giorgino, "Free triiodothyronine and thyroid stimulating hormone are directly associated with waist circumference, independently of insulin resistance, metabolic parameters and blood pressure in overweight and obese women," Clinical Endocrinology, vol. 67, no. 2, pp. 265-269, 2007.

[42] T. Reinehr, "Obesity and thyroid function," Molecular and Cellular Endocrinology, vol. 316, no. 2, pp. 165-171, 2010.

[43] T. Reinehr, G. de Sousa, and W. Andler, "Hyperthyrotropinemia in obese children is reversible after weight loss and is not related to lipids," Journal of Clinical Endocrinology and Metabolism, vol. 91, no. 8, pp. 3088-3091, 2006.

[44] S. Shalitin, M. Yackobovitch-Gavan, and M. Phillip, "Prevalence of thyroid dysfunction in obese children and adolescents before and after weight reduction and its relation to other metabolic parameters," Hormone Research, vol. 71, no. 3, pp. 155-161, 2009.

[45] T. Reinehr and W. Andler, "Thyroid hormones before and after weight loss in obesity," Archives of Disease in Childhood, vol. 87, no. 4, pp. 320-323, 2002.

[46] L. H. Duntas and L. Wartofsky, "Cardiovascular risk and subclinical hypothyroidism: focus on lipids and new emerging risk factors. What is the evidence?" Thyroid, vol. 17, no. 11, pp. 1075-1084, 2007.

[47] N. Rodondi, D. Aujesky, E. Vittinghoff, J. Cornuz, and D. C. Bauer, "Subclinical hypothyroidism and the risk of coronary heart disease: a meta-analysis," The American Journal of Medicine, vol. 119, no. 7, pp. 541-551, 2006.

[48] J. P. Walsh, "Setpoints and susceptibility: do small differences in thyroid function really matter?" Clinical Endocrinology, vol. 75, no. 2, pp. 158-159, 2011. 


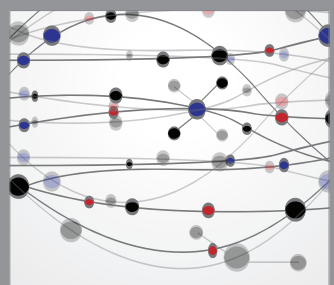

The Scientific World Journal
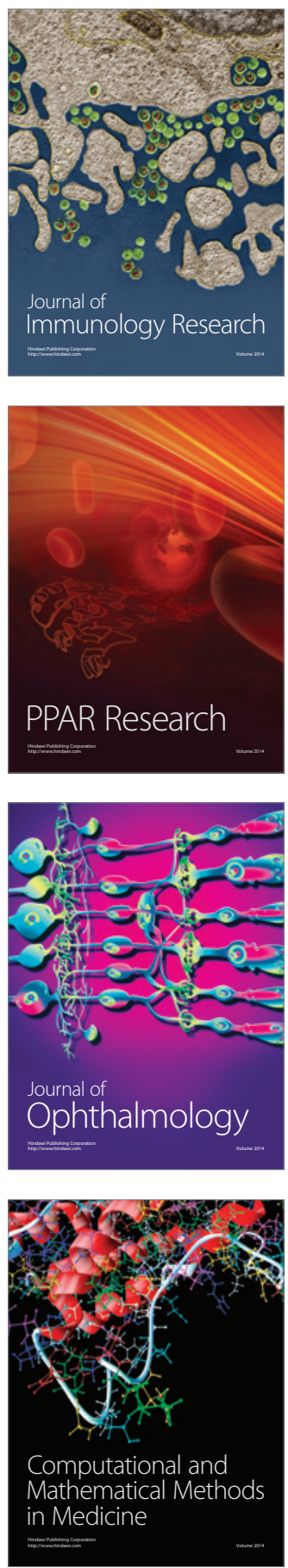

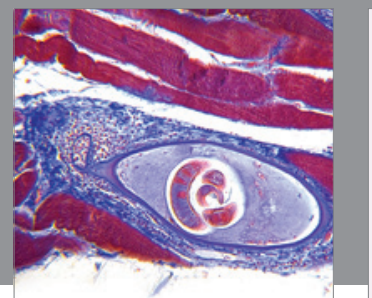

Gastroenterology

Research and Practice
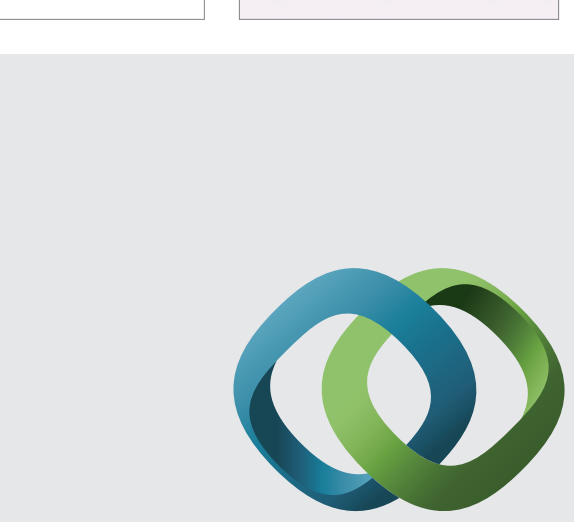

\section{Hindawi}

Submit your manuscripts at

http://www.hindawi.com
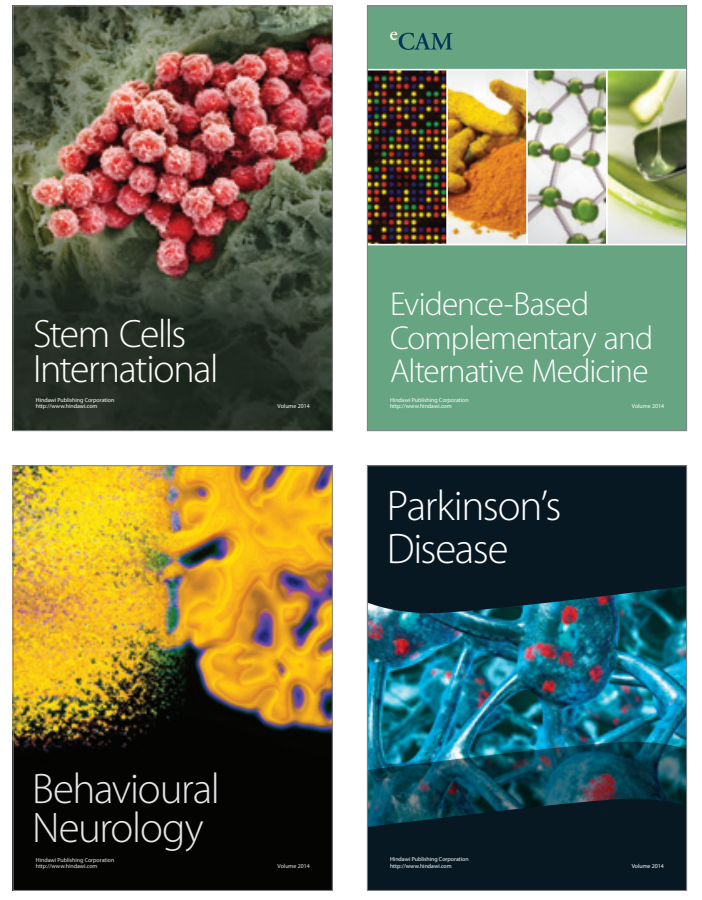
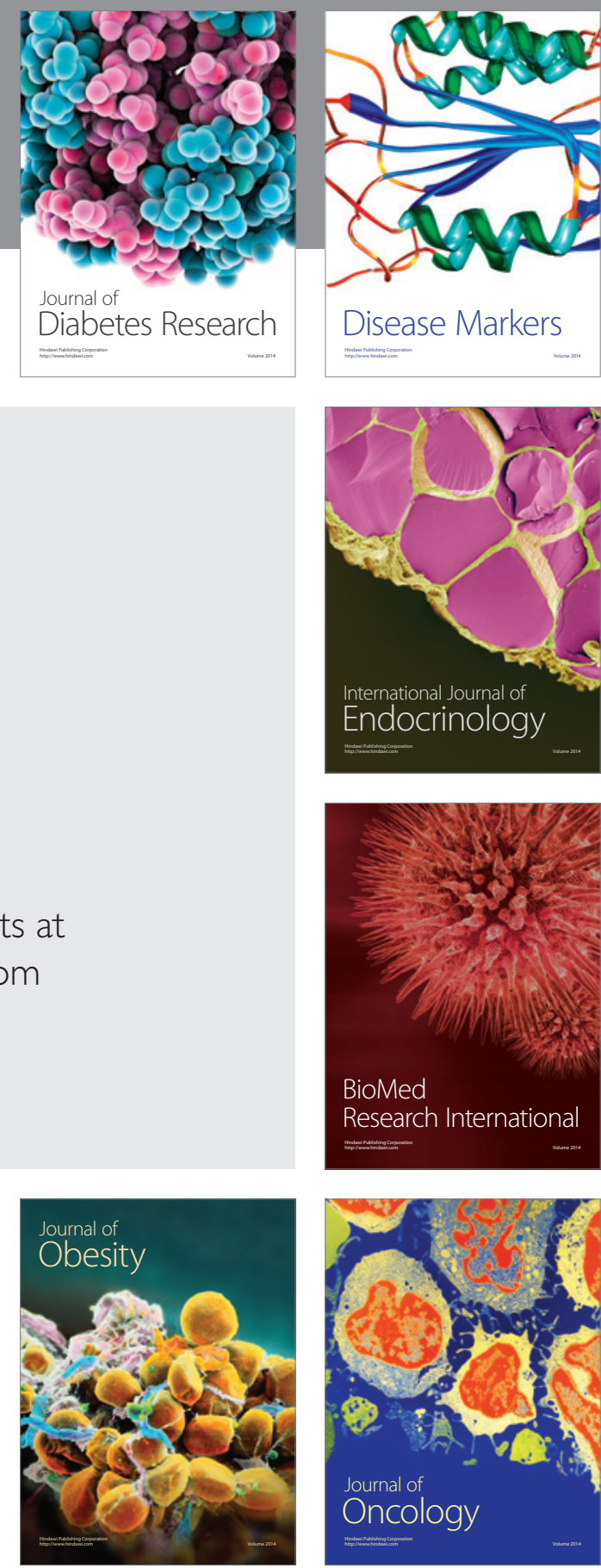

Disease Markers
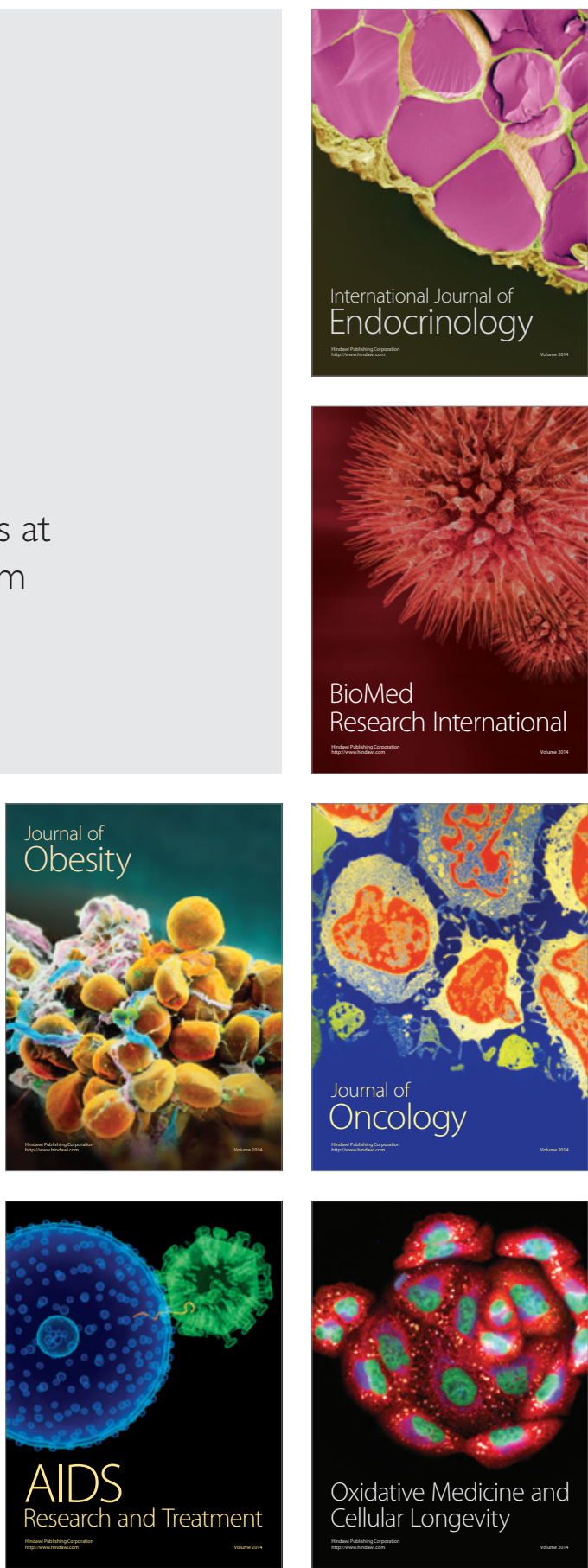